Fluctuation and Noise Letters

Vol. 0, No. 0 (2005) 000-000

(C) World Scientific Publishing Company

\title{
ROLE OF THE COLORED NOISE IN SPATIO-TEMPORAL BEHAVIOR OF TWO COMPETING SPECIES
}

\author{
D. Valenti ${ }^{\circ}$, A. Fiasconaro and B. Spagnolo \\ Dipartimento di Fisica e Tecnologie Relative and INFM, Group of Interdisciplinary Physics* \\ Università di Palermo, Viale delle Scienze pad. 18, I-90128 Palermo, Italy \\ ${ }^{\circ}$ valentid@gip.dft.unipa.it \\ Received (15 January 2005) \\ Revised (23 April 2005) \\ Accepted (6 May 2005) \\ Communicated by Werner Ebeling and Bernardo Spagnolo
}

\begin{abstract}
We study the spatial distributions of two randomly interacting species, in the presence of an external multiplicative colored noise. The dynamics of the ecosystem is described by a coupled map lattice model. We find a nonmonotonic behavior in the formation of large scale spatial correlations as a function of the multiplicative colored noise intensity. This behavior is shifted towards higher values of the noise intensity for increasing correlation time of the noise.

Keywords: Statistical mechanics; noise induced effects; Lotka-Volterra equations; spatiotemporal patterns.
\end{abstract}

\section{Introduction}

Recently there is an increasing research activity on the role of noise in spatially extended systems [1, 2, 3]. One of the challenging problems to be understood is the formation of large scale spatial correlations. In this work we present a theoretical investigation on the role played by the colored noise in the transient dynamics of the spatial distributions of two competing species. The colored noise in spatially extended systems has been investigated in the past [2] (and references cited there), 4. Aim of the work is to present a stochastic model with a realistic noise and to suggest a possibility of application to biological systems, where the presence of fluctuations, such as random variability of temperature, can modify strongly the dynamics of an ecosystem [5, 6, 7 . The theoretical results in fact may be compared with the experimental data obtained for the biomass of species measured in the Sicily Channel [8]. Our investigation could contribute (i) to select environmental forcings which affect the dynamics of biological systems [6, 9, 10, and (ii) to implement physical models for the interpretation of spatial distributions of marine species.

*Electronic address: http://gip.dft.unipa.it 


\section{The Model}

To study the colored noise effect on the formation of large scale spatial correlations of two species densities, we consider a coupled map lattice model [11,12]

$$
\begin{aligned}
x_{i, j}^{n+1} & =\gamma x_{i, j}^{n}\left(1-x_{i, j}^{n}-\beta^{n} y_{i, j}^{n}\right)+x_{i, j}^{n}\left(\zeta_{i, j}^{n}\right)_{x}+D \sum_{\delta}\left(x_{\delta}^{n}-x_{i, j}^{n}\right), \\
y_{i, j}^{n+1} & =\gamma y_{i, j}^{n}\left(1-y_{i, j}^{n}-\beta^{n} x_{i, j}^{n}\right)+y_{i, j}^{n}\left(\zeta_{i, j}^{n}\right)_{y}+D \sum_{\delta}\left(y_{\delta}^{n}-y_{i, j}^{n}\right),
\end{aligned}
$$

where $x_{i, j}^{n}$ and $y_{i, j}^{n}$ are the densities of two species in the site $(i, j)$ at the time step $n, \gamma$ is proportional to the growth rate, $D$ is the diffusion coefficient, $\sum_{\delta}$ is the sum over the four nearest neighbors, and $\beta^{n}$ is the interaction parameter. Here $\left(\zeta_{i, j}^{n}\right)_{x}$ and $\left(\zeta_{i, j}^{n}\right)_{y}$ are Ornstein-Uhlenbeck colored noises at each site $(i, j)$, and correspond to the value of the stochastic continuous process $\zeta_{l}(t)$ given by the equation

$$
\frac{d \zeta_{l}(t)}{d t}=-\frac{1}{\tau_{c}} \zeta_{l}(t)+\frac{1}{\tau_{c}} \xi_{l}(t), \quad(l=x, y)
$$

taken at the time step $n$. Here $\xi_{i}(t)(i=x, y)$ are Gaussian white noises within the Ito scheme with zero mean and correlation function $\left\langle\xi_{l}(t) \xi_{k}\left(t^{\prime}\right)\right\rangle=\sigma \delta\left(t-t^{\prime}\right) \delta_{l k}$. The correlation function of the processes of Eq. (3) is

$$
\left\langle\zeta_{l}(t) \zeta_{k}\left(t^{\prime}\right)\right\rangle=\frac{\sigma}{2 \tau_{c}} e^{-\left|t-t^{\prime}\right| / \tau_{c}} \delta_{l k}
$$

and gives $\sigma \delta\left(t-t^{\prime}\right) \delta_{l k}$ in the limit $\tau_{c} \rightarrow 0$. In absence of noise, for $\beta<1$ both species survives in a coexistence regime, while for $\beta>1$ one of the two species vanishes after a certain time and an exclusion regime takes places. To describe the noisy interaction between species and the environment we consider an Ito stochastic differential equation for the parameter $\beta$ with a periodical driving force [12,13, 14.

$$
\frac{d \beta(t)}{d t}=-\frac{d U(\beta)}{d \beta}+\operatorname{acos}\left(\omega_{0} t\right)+\xi_{\beta}(t) .
$$

Here $U(\beta)$ describes the two stable states of the ecological system (coexistence and exclusion)

$$
U(\beta)=h(\beta-(1+\rho))^{4} / \eta^{4}-2 h(\beta-(1+\rho))^{2} / \eta^{2} .
$$

The values of the parameters $h, \rho$ and $\eta$ depend on intrinsic biological characteristics of the species, as stability of their life cycles respect to external perturbations and ability of one species to compensate the increase or decrease of the other species. The periodical driving mimics the climatic temperature oscillations, and $\xi_{\beta}(t)$ is a white Gaussian noise with $\left\langle\xi_{\beta}(t)>=0\right.$ and $<\xi_{\beta}(t) \xi_{\beta}\left(t^{\prime}\right)>=\sigma_{\beta} \delta\left(t-t^{\prime}\right)$. The interaction parameter $\beta^{n}$ of Eqs. (1), (2) corresponds to the value of the stochastic process $\beta(t)$ of Eq. (15) taken at the time step $n$. In the absence of the additive noise, $\beta(t)$ oscillates periodically and its values always remain below $\beta=1$, giving rise to a coexistence regime. We fix the additive noise intensity at the value $\sigma_{\beta}=2.65 \cdot 10^{-3}$ corresponding to a competition regime with $\beta$ periodically switching from coexistence to exclusion regions. This produces the appearance of stochastic resonance 
(SR) effect in the dynamics of interaction parameter $\beta$ and noise-induced anticorrelated periodic oscillations in the time evolution of the two species densities [12]14]. We analyze the spatial distribution of the ecosystem in this SR dynamical regime, by varying both the intensities of multiplicative colored noise in Eqs. (1) and (2).

\subsection{Spatial distributions}

By using a square spatial lattice with $N=100 \times 100$ sites, and species densities vanishing on the grid borders, we obtain the spatio-temporal distributions of the two species for uniform initial condition and for three values of the correlation time $\tau_{c}$, namely $\tau_{c}=10^{-2}, 10,10^{2}$. We find that for the lowest value of the correlation time $\left(\tau_{c}=10^{-2}\right)$, the spatial distribution is almost the same as that found for multiplicative white noise 12. In Fig. [1 the spatial distributions of the two species

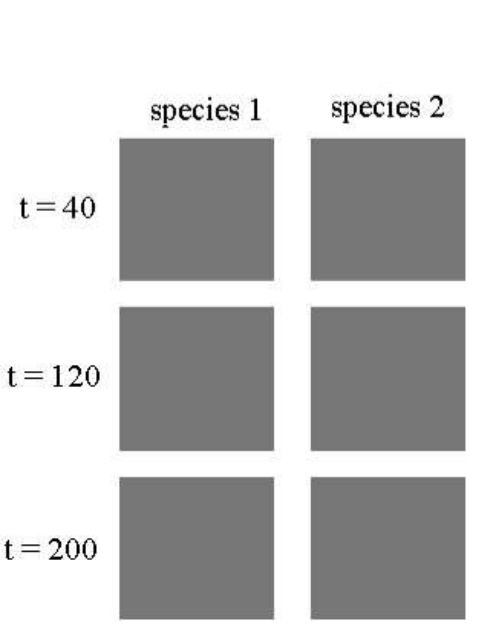

(a)

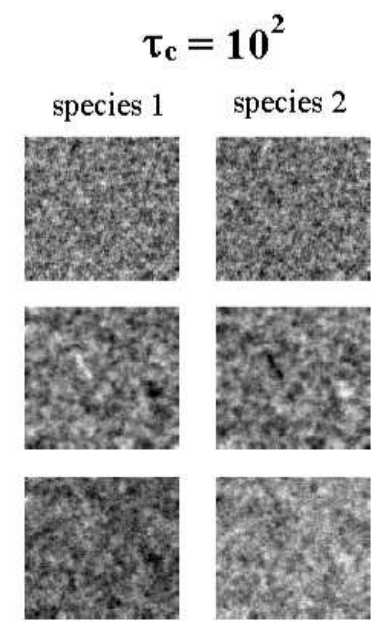

(b)

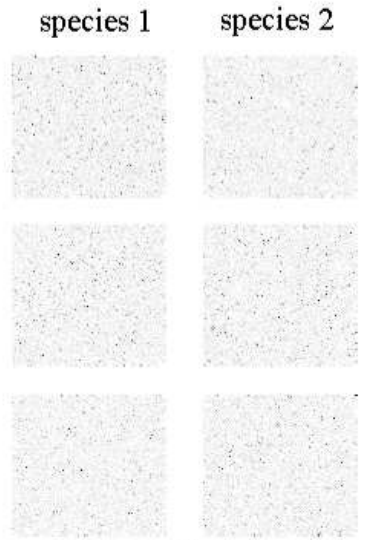

(c)

Fig 1. Spatial distributions with $\tau_{c}=10^{2}$ at different times for (a) $\sigma=10^{-12}$, (b) $\sigma=10^{-4}$, (c) $\sigma=10^{5}$. The values of the parameter set are: $\sigma_{\beta}=2.65 \cdot 10^{-3}, \gamma=2, D=0.05, a=1.5 \cdot 10^{-1}$, $\omega_{0} /(2 \pi)=10^{-2}, \beta(0)=0.94, N=100 \times 100$, and $x_{i, j}^{0}=y_{i, j}^{0}=0.5$ at all sites $(i, j)$.

are obtained with correlation time $\tau_{c}=10^{2}$ and for $\sigma=10^{-12}, 10^{-4}, 10^{5}$. We see that for very low noise intensity (Fig. प(a)) the spatial distributions of the species are uniform as at $t=0$. For higher noise intensities (see Fig. 1(b)) an anticorrelation between the two species is observed: the two species tend to occupy different positions. Further increase of the noise causes the anticorrelation to disappear and the two species densities become uncorrelated (see Fig. 1(c)). We observe that anticorrelated behavior is stronger for increasing $\tau_{c}$. In particular by comparing the distributions obtained with different values of correlation time $\tau_{c}$ we note that for $\tau_{c}=10^{2}$ the two species densities are strongly anticorrelated. This is expressed by the presence, in the same spatial region, of a high density (dark zones) of species 1 (species 2) corresponding to a low density (light zones) of species 2 (species 1) (see Figs. 1(b)). 


\section{2. $\quad$ Correlation between species}

To analyze the role of the colored noise on the correlation between the two species, we calculate for different values of the correlation time $\tau_{c}$, at the time step $n$, the correlation coefficient $c^{n}$ defined on the lattice as

$$
c^{n}=\frac{\operatorname{cov}_{x y}^{n}}{s_{x}^{n} s_{y}^{n}}
$$

with

$$
\operatorname{cov}_{x y}^{n}=\frac{\sum_{i, j}\left(x_{i, j}^{n}-\bar{x}^{n}\right)\left(y_{i, j}^{n}-\bar{y}^{n}\right)}{N},
$$

where $\bar{x}^{n}, s_{x}^{n}, \bar{y}^{n}, s_{y}^{n}$ are the mean value and the root mean square respectively of species 1 and species 2, obtained over the whole spatial grid at the time step $n$, $\operatorname{cov}_{x y}^{n}$ is the corresponding covariance and $N=100 \times 100$ is the number of sites of the considered lattice. The behavior of the correlation coefficient $c^{n}$ as a function of time has been obtained for $\tau_{c}=10^{-2}, 10,10^{2}$, and for different levels of the multiplicative colored noise. For $\sigma=0$ the species are strongly correlated and $c^{n}$ is constant. In Fig. 2 we report the behavior of $c^{n}$ for $\tau_{c}=10^{-2}$. For low levels of

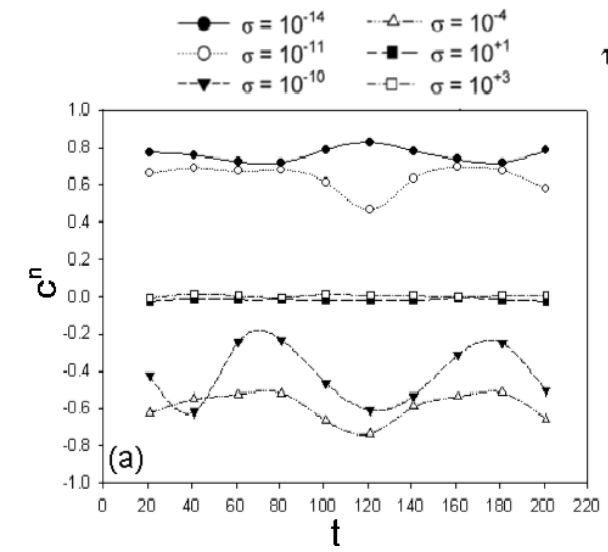

$$
\tau_{\mathrm{c}}=10^{-2}
$$

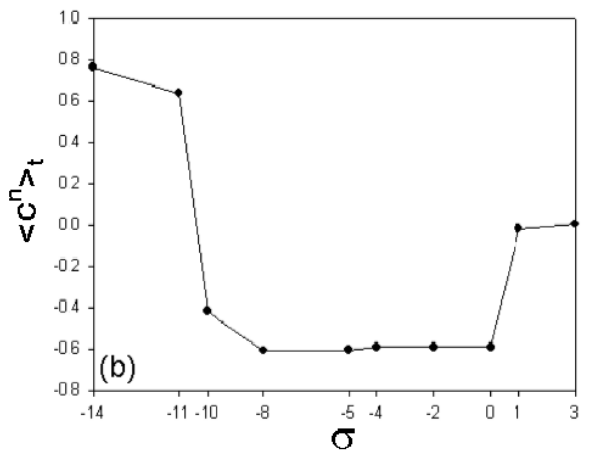

Fig 2. (a) Correlation coefficient $c^{n}$ as a function of time, for $\tau_{c}=10^{-2}$ and for different values of the multiplicative noise intensity $\sigma$, namely $\sigma=10^{-14}, 10^{-11}, 10^{-10}, 10^{-4}, 10^{+1}, 10^{+3}$; (b) time average correlation coefficient $\left\langle c^{n}\right\rangle_{t}$ as a function of the multiplicative noise intensity.

the multiplicative colored noise $\left(\sigma=10^{-14}, \sigma=10^{-11}\right)$, the values of $c^{n}$ oscillate between 0.5 and 0.8 , showing a stronger correlation between species for lower values of $\sigma$. For intermediate values of noise intensity, namely for $\sigma=10^{-10}, 10^{-4}$ the spatial correlation coefficient $c_{n}$ oscillates between -0.6 and -0.2 at the frequency of the periodical forcing, showing strong anticorrelation between the two species. By increasing the noise intensity, the anticorrelation is reduced and finally it disappears $\left(\sigma=10^{+1}, \sigma=10^{+3}\right)$, that is the species are totally uncorrelated. A nonmonotonic behavior of the correlation coefficient as a function of the multiplicative intensity appears clearly. This behavior is more evident by considering the time average correlation coefficient $\left\langle c^{n}\right\rangle_{t}$ as a function of the multiplicative noise intensity (see Fig. 2(b)). The minimum in Fig. 2(b) corresponds to the anticorrelated oscillations 
shown in Fig. 2(a). Moreover this behavior is connected with the anticorrelated oscillations present in the time evolution of two competing species in each point of our spatial grid 14. By comparing the results shown in Fig. 2 with the white noise case [12] we observe that the effect of a small correlation time $\tau_{c}$ is to produce an enlargement of the minimum of $\left\langle c^{n}\right\rangle_{t}$ towards higher values of the multiplicative noise intensity. The ecosystem remains in his anticorrelated regime for a longer time due to the memory of the colored noise. The two noise sources have different roles
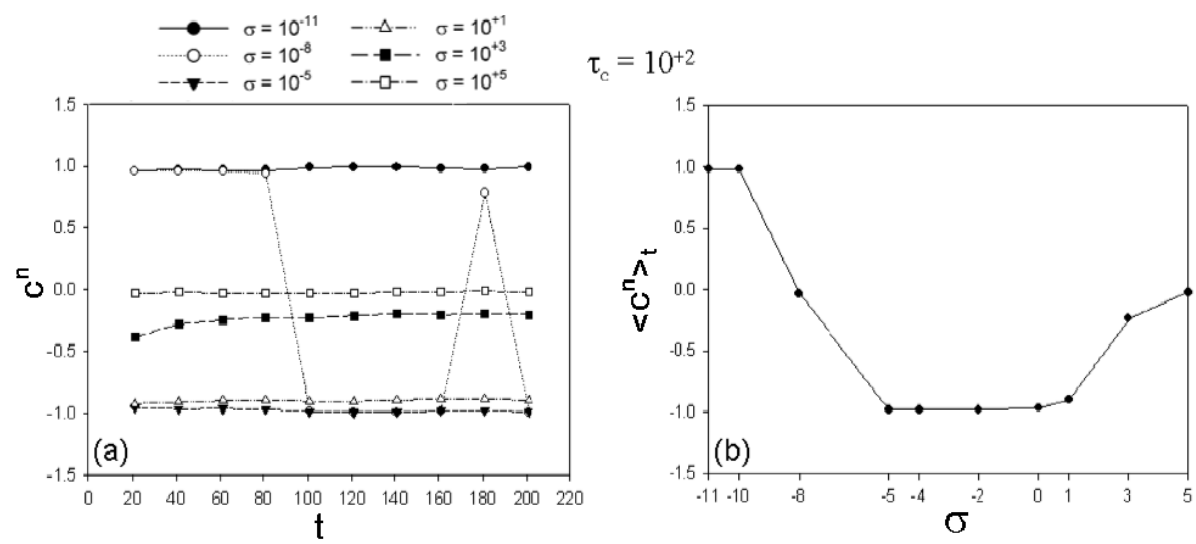

Fig 3. (a) Correlation coefficient $c^{n}$ as a function of time, for $\tau_{c}=10^{2}$ and for different values of the multiplicative noise intensity $\sigma$, namely $\sigma=10^{-11}, 10^{-8}, 10^{-5}, 10^{+1}, 10^{+3}, 10^{+5}$; (b) time average correlation coefficient $\left\langle c^{n}\right\rangle_{t}$ as a function of the multiplicative noise intensity.

on the dynamics of our ecosystem. Specifically the additive noise affects directly the dynamical regime, while the multiplicative noise breaks the symmetric dynamical behavior of the two species by producing a coherent response of the system [12 14, which is responsible for the appearance of anticorrelation in the spatial distributions of the two species. In Fig. 3 the behavior of $c^{n}$ and $\left\langle c^{n}\right\rangle_{t}$ is reported for $\tau_{c}=10^{2}$. As in the previous case we observe a nonmonotonic behavior of $c^{n}$ as a function of the multiplicative noise intensity. For low noise intensities $\left(\sigma=10^{-11}, \sigma=10^{-10}\right)$, the species are strongly correlated and $c^{n}$ is almost constant. For $\sigma=10^{-8}$ strong oscillations between +1.0 and -1.0 appear. This effect is due to the colored noise, in fact the ecosystem remains periodically in the correlated or anticorrelated regime for a time interval of the order of the correlation time of the noise. For greater noise intensity $\left(\sigma=10^{-5}, 10^{1}\right)$, the anticorrelation regime takes place $\left(c^{n} \approx-1.0\right)$. By increasing the noise intensity $\left(\sigma=10^{+3}, 10^{+5}\right), c^{n}$ goes through a nonmonotonic behavior, taking on values around zero (uncorrelated regime). Comparing Fig. 2 and Fig. 3] we observe that the presence of a bigger correlation time $\tau_{c}$ determines a stronger anticorrelation in the two species distributions. The minimum of the time average correlation time $\left\langle c^{n}\right\rangle_{t}$ is deeper and it is shifted towards higher values of the multiplicative noise intensity for greater values of correlation time $\tau_{c}$. A highly correlated noise therefore produces two effects: (i) the system is pushed towards a strongly anticorrelated regime $\left(c^{n} \approx-1.0\right)$; (ii) this regime takes place for higher values of multiplicative noise intensity. The colored noise produces therefore a persistence of the dynamical regime experienced by the ecosystem. 


\section{Conclusions}

In this work we analyze the effects of the colored noise in the spatial distributions of two competing species. By using a discrete time evolution model, which is the discrete version of the Lotka-Volterra equations with diffusive terms, in the presence of a multiplicative colored noise, with a random interaction parameter, we analyze the spatio-temporal behavior of the two species. We find that the colored noise determines an enhancement of the effects observed in the presence of white noise [12], that is (i) formation of large scale spatial correlations with the same periodicity of the deterministic force and (ii) anticorrelation in the spatial distributions of the two species. Finally we observe that (i) the intensity of the anticorrelation increases as a function of $\tau_{c}$, (ii) the system remains in the anticorrelated region for a bigger range of multiplicative noise values as $\tau_{c}$ increases. The noise spectrum of a real physical system is characterized by a cut-off (correlated noise). Therefore data from real ecosystems, whose dynamics is strongly affected by fluctuations and by environmental physical variables, can be modeled using sources of colored noise.

\section{Acknowledgements}

This work has been supported by INTAS Grant 01-450, INFM and MIUR.

\section{References}

[1] A. A. Zaikin and L. Schimansky-Geier, Spatial patterns induced by additive noise, Phys. Rev. E 58 (1998) 4355-4360.

[2] J. García-Ojalvo and J. M. Sancho, Noise in Spatially Extended Systems, SpringerVerlag, NY (1999).

[3] M. Ibanes, J. M. Sancho, J. Buceta and K. Lindenberg, Noise-driven mechanism for pattern formation, Phys. Rev. E 67 (2003) 021113-(1-8).

[4] J. García-Ojalvo and J. M. Sancho, Colored noise in spatially extended systems, Phys. Rev. E 49 (1994) 2769-2778.

[5] C. Zimmer, Life After Chaos, Science 284 (1999) 83-86.

[6] O. N. Bjørnstad and B. T. Grenfell, Noisy clockwork: Time series analysis of population fluctuations in animals, Science 293 (2001) 638-643.

[7] S. Ciuchi, F. de Pasquale and B. Spagnolo, Self regulation mechanism of an ecosystem in a non gaussian fluctuation regime, Phys. Rev. E 53 (1996) 706-716.

[8] J. García Lafuente, A. García, S. Mazzola, L. Quintanilla, J. Delgado, A. Cuttitta and B. Patti, Hydrographic phenomena influencing early life stages of the Sicilian Channel anchovy, Fisheries Oceanography 11/1 (2002) 31-44.

[9] O. N. Bjørnstad, J. M. Fromentin, N. C. Stenseth and J. Gjøsæter, Cycles and trends in cod populations, Proc. Natl. Acad. Sci. USA 96 (1999) 5066-5071.

[10] R. B. Alley, S. Anandakrishnan and P. Jung, Stochastic resonance in the North Atlantic, Paleoceanography 16 (2001) 190-198.

[11] Special issue CML models, edited by K. Kaneko [Chaos 2 279-460 (1992)].

[12] D. Valenti, A. Fiasconaro and B. Spagnolo, Pattern formation and spatial correlation induced by the noise in two competing species, Acta Phys. Pol. B 35 (2004) 1481-1489.

[13] B. Spagnolo, M. Cirone, A. La Barbera and F. de Pasquale, Noise induced effects in population dynamics, J. Physics: Cond. Matter 14 (2002) 2247-2255.

[14] D. Valenti, A. Fiasconaro and B. Spagnolo, Stochastic resonance and noise delayed extinction in a model of two competing species, Physica A 331 (2004) 477-486. 